\title{
Effect of Equilibration on Primitive Path Analyses of Entangled Polymers
}

\author{
Robert S. Hoy* and Mark O. Robbins \\ Department of Physics and Astronomy, Johns Hopkins University, Baltimore, Maryland 21218
}

(Dated: October 22, 2005)

\begin{abstract}
We use recently developed primitive path analysis (PPA) methods to study the effect of equilibration on entanglement density in model polymeric systems. Values of $N_{e}$ for two commonly used equilibration methods differ by a factor of two to four even though the methods produce similar large-scale chain statistics. We find that local chain stretching in poorly equilibrated samples increases entanglement density. The evolution of $N_{e}$ with time shows that many entanglements are lost through fast processes such as chain retraction as the local stretching relaxes. Quenching a melt state into a glass has little effect on $N_{e}$. Equilibration-dependent differences in short-scale structure affect the craze extension ratio much less than expected from the differences in PPA values of $N_{e}$.
\end{abstract}

PACS numbers: 83.10.Kn,82.35.Lr,02.70-c,61.41.+e

\section{INTRODUCTION}

Much of the physics of bulk polymeric material is independent of chemical detail and arises instead from the connectivity of the constituent chain molecules, provided the material is sufficiently dense and the chains sufficiently long. Since chains can not pass through each other, they are confined to "tubes" by topological constraints that are often associated with discrete "entanglements". The tube model of Edwards and de Gennes has been a focus of extensive study over the past few decades [1, 2] and qualitatively describes many aspects of entangled polymer dynamics. Perhaps its most famous prediction is the relation $\eta \propto N^{3}$ for $N \gg N_{e}$, where $\eta$ is the melt viscosity, $N$ is the degree of polymerization, and $N_{e}$ is the "entanglement length".

A key construct of the tube model is the "primitive path", the shortest contour into which a chain molecule fixed at its ends may contract without crossing any other chains [1]. Everaers and collaborators have recently used this construct to perform "primitive path analysis" (PPA) of model entangled polymeric systems [3, 4]. Their results for $N_{e}$ agree with the predictions of a chain packing model [5] that explains trends in experimental results for melts and semidilute solutions of linear homopolymers [5, 6, 7, 8, 9]. The PPA technique is a promising tool for obtaining information about the behavior of individual entanglements which has not been accessible through other theoretical or experimental means.

In this paper, we address the relation of melt equilibration to the entanglement length measured by PPA analysis. Experimental studies of linear homopolymer melts have shown that $N_{e}$ is controlled by the Gaussian statistics of chains at large scales [5, 6, 6]. One might reasonably assume that values of $N_{e}$ from PPA analysis of model melts would also be controlled by large-scale chain structure. Our results, however, show that this assumption can fail badly. Well-equilibrated states pro-

*Electronic address: robhoy@pha.jhu.edu duced using a double-bridging algorithm 10 are compared to states produced using the common fast-pushoff method. For typical equilibration times, use of the fast pushoff produces chain stretching at short scales while preserving large-scale structure [10]. This stretching can produce values of $N_{e}$ that are two to four times smaller than those for well-equilibrated states, showing that failure to accurately equilibrate short-scale chain structure can cause large systematic errors in PPA analyses.

Our values of $N_{e}$ for the well-equilibrated states agree with values from previous PPA analyses 3, 4, 11] and from other methods [12]. We verify that the reduction of $N_{e}$ for poorly equilibrated states reflects a real excess of topological constraints, and show that early stages of the evolution of $N_{e}$ towards equilibrium can occur through fast topology-changing processes familiar from tube theories 2], such as cooperative chain retraction and constraint release. The changes in $N_{e}$ with improving equilibration are correlated to changes in chain structure.

We also perform PPA analyses of glassy states. In interpreting the mechanical properties of polymer glasses, it is often assumed that the value of $N_{e}$ in the glass is inherited from the melt [13, 14]. While PPA values for $N_{e}$ drop slightly upon cooling from a melt to a glass well below $T_{g}$, the changes are comparable to systematic and statistical uncertainties. Glass values for $N_{e}$ can not be compared to rheological measurements, but can be obtained from the extension ratio during craze formation [14, 15]. We find that this measure of $N_{e}$ is less sensitive to equilibration than PPA values, perhaps because deformation into the craze structure removes excess entanglements produced by local stretching.

\section{POLYMER MODEL: METHODS OF MELT EQUILIBRATION}

We employ a coarse-grained bead-spring polymer model [16, 17] that incorporates key physical features of linear homopolymers such as covalent backbone bonds, excluded-volume interactions, chain stiffness, and the topological restriction that chains may not cross. All 
monomers have mass $m$ and interact via the truncated and shifted Lennard-Jones potential:

$$
U_{L J}(r)=4 \epsilon\left(\left(\left(\frac{a}{r}\right)^{12}-\left(\frac{a}{r_{c}}\right)^{12}\right)-\left(\left(\frac{a}{r}\right)^{6}-\left(\frac{a}{r_{c}}\right)^{6}\right)\right),
$$

where $r_{c}$ is the potential cutoff radius and $U_{L J}(r)=0$ for $r>r_{c}$. Unless noted, $r_{c}=2^{1 / 6} a$. We express all quantities in terms of length $a$, energy $\epsilon$, and time $\tau_{L J}=$ $\sqrt{m a^{2} / \epsilon}$.

Covalent bonds between adjacent monomers on a chain are modeled using the finitely extensible nonlinear elastic (FENE) potential

$$
U_{F E N E}(r)=-\frac{k R_{0}^{2}}{2} \ln \left(1-\left(\mathrm{r} / \mathrm{R}_{0}\right)^{2}\right),
$$

with the canonical parameter choices $R_{0}=1.5 a$ and $k=$ $30 \epsilon / a^{2}$ 17]. The chains contain $N$ monomers and have an equilibrium bond length $l_{0}=.96 a$. Chain stiffness is modeled using the potential

$$
\begin{aligned}
U_{\text {bend }}(r) & =k_{\text {bend }}\left(1-\frac{\vec{b}_{i-1} \cdot \vec{b}_{i}}{\left|\vec{b}_{i-1}\right|\left|\vec{b}_{i}\right|}\right) \\
& =k_{\text {bend }}\left(1-\cos \left(\theta_{i}\right)\right)
\end{aligned}
$$

where $\vec{r}_{i}$ denotes the position of the ith monomer on a chain and $\theta_{i}$ is the angle betweeen consecutive bond vectors $\vec{b}_{i-1}=\vec{r}_{i}-\vec{r}_{i-1}$ and $\vec{b}_{i}=\vec{r}_{i+1}-\vec{r}_{i}$.

Newton's equations of motion are integrated with the velocity-Verlet method [18 and timestep $\delta t=.005 \tau_{L J}-$ $.012 \tau_{L J}$. The system is coupled to a heat bath at temperature $T$ using a Langevin thermostat [19]. Unless noted, the simulation cell is a cube whose size is chosen so that the monomer number density is $\rho=0.85 a^{-3}$. Periodic boundary conditions are applied in all three directions. At this density and $T=1.0 \epsilon / k_{B}$ the system is a melt well above the glass transition [17]. For this model, published entanglement lengths vary from about 70 for fully flexible chains $\left(k_{\text {bend }}=0\right)$ to 20 for semiflexible chains with $k_{\text {bend }}=2.0 \epsilon[3,4,11,12,15,20$. For the chain lengths used in this study $(N=350$ and $N=500)$, larger values of $k_{\text {bend }}$ produce nematic order [21].

We contrast two common methods for creating initial states for the PPA analysis. Both begin by generating initial configurations of $N_{c h}$ chains without considering excluded volume 22]. Each initial chain configuration is a random walk of $N-1$ steps with the bond angles chosen to give the desired chain statistics [10]

$$
\begin{aligned}
& <R^{2}(n)>=n l_{0}^{2}\left(\frac{1+<\cos (\theta)>}{1-<\cos (\theta)>}\right. \\
& \left.-\frac{2}{n} \frac{<\cos (\theta)>\left(1-<\cos (\theta)>^{n}\right)}{(1-<\cos (\theta)>)^{2}}\right),
\end{aligned}
$$

where $<R^{2}(n)>$ is the average squared distance between monomers separated by chemical distance $n$. Large scale measures of chain structure such as the chain stiffness constant $C_{\infty}$ and Kuhn length $l_{K}$ are related to the chain statistics by

$$
C_{\infty}=\frac{l_{K}}{l_{0}}=\frac{1+<\cos (\theta)>}{1-<\cos (\theta)>} .
$$

Lennard-Jones interactions for nonbonded monomers cannot be introduced immediately after creating the initial chain configurations because chains spatially overlap 222. Instead, a soft repulsive potential is used to reduce the chain overlap through gradual introduction of excluded volume interactions. The specific soft potential used is

$$
U_{\text {soft }}(r)=\left\{\begin{array}{cc}
A\left[1+\cos \left(\frac{\pi r}{2^{1 / 6} a}\right)\right] & , r<2^{1 / 6} a \\
0 & , r>2^{1 / 6} a
\end{array}\right.
$$

for nonbonded beads. The value of A is linearly increased from $4 \epsilon$ to $100 \epsilon$ over a time $20 \tau_{L J}$, as in Ref. [10. Unfortunately, this "fast pushoff" procedure creates significant distortions in the chain statistics on length scales comparable to the tube diameter [10, 23]. Chains are stretched well beyond the form of Eqn. (4) at intermediate chemical distances [10].

After the fast pushoff is completed, the two methods of equilibration differ. In the pure molecular dynamics (PMD) method, normal Lennard-Jones interactions are activated, and integration is continued for up to $4.5 \cdot 10^{5} \tau_{L J}$. This maximum equilibration time is still much shorter than the longest relaxation time of the systems we consider, which is the disentanglement time $\tau_{d}$. Only after an equilibration run of more than $\tau_{d}$ do the chain configurations come to full equilibrium [10], but equilibration runs much shorter than $\tau_{d}$ are commonly used in PMD simulations 10, 17]. We therefore refer to our PMD-prepared states as "poorly equilibrated".

The other equilibration method used is the doublebridging-MD hybrid (DBH) algorithm described in Ref. [10]. In addition to standard MD equilibration, Monte Carlo moves which alter the connectivity of chain subsections are periodically performed, allowing the chain configurations to relax far more rapidly 24]. Equilibrated chain statistics from extremely long MD runs were used to obtain target functions for these Monte Carlo moves 10, 12]. We therefore refer to the DBH-prepared states as "well equilibrated".

If a glassy state is desired, we increase $r_{c}$ to $1.5 a$ and perform a rapid temperature quench at a cooling rate of $\dot{T}=-2 \times 10^{-3}\left(\epsilon / k_{B}\right) / \tau_{L J}$. The systems are cooled to $T=.55 \epsilon / k_{B}$ at constant density, and then to $T=.1 \epsilon / k_{B}$ at zero pressure using a Nose-Hoover barostat 18. The resulting glasses have density $\rho \simeq 1.02 a^{-3}$. Other temperature and pressure protocols give similar results for $N_{e}$.

Once preparation of the system is complete, we perform primitive path analyses as in Refs. 3, 4]. Except as noted in Section IIII the procedure is nearly identical to that of Ref. [4], and we refer to it as the "standard" PPA procedure. All chain ends are fixed in space and several 
changes are made to the interaction potential. Intrachain excluded-volume interactions are deactivated, while interchain excluded-volume interactions are retained. The covalent bonds are strengthened by setting $k=100 \epsilon$, and the bond lengths are capped at $1.2 a$ to prevent chains from crossing one another [4]. Note that we do not attempt to preserve self-entanglements, since their number is negligibly small for the systems considered here [4]. For semiflexible chains, the bond-bending potential is deactivated by setting $k_{\text {bend }}=0$. The system is then coupled to a heat bath at $T=.001 \epsilon / k_{B}$ so that thermal fluctuations are negligible, and the equations of motion are integrated until the chains minimize their length. This typically requires from 500 to $2000 \tau_{L J}$. Other variants of the PPA procedure are discussed in Section IIA

Once the chain contour lengths have been minimized, we use the formula given in Ref. 3] to calculate the entanglement length:

$$
N_{e}=\frac{<R_{e e}^{2}>}{(N-1)<b_{p p}>^{2}},
$$

where $\left\langle R_{e e}^{2}\right\rangle$ is the average squared end-end distance, and $\left\langle b_{p p}\right\rangle$ is the mean bond length at the end of the PPA run [3]. We also calculate the rms variation of $b_{p p}$ for each chain, and report the mean of this quantity as $\Delta b_{p p}$. An alternative method for calculating $N_{e}$ is to fit the primitive path chain statistics to Eqn. (4), with $l_{0}$ and $\langle\cos (\theta)\rangle$ as fitting parameters [4]. The fit value of $\langle\cos (\theta)\rangle$ is then inserted into Eqn. [5] and $N_{e}$ is identified with $C_{\infty}$ [4]. In every case this gave values of $N_{e}$ consistent with the values from Eqn. (7), confirming that the primitive paths have Gaussian random walk statistics, with $N_{e}$ monomers per Kuhn segment [3].

\section{RESULTS}

\section{A. Dependence of $N_{e}$ on Preparation Method}

Table \ shows results from PPA runs for flexible $\left(k_{\text {bend }}=0\right)$ and semiflexible $\left(k_{\text {bend }}=0.75 \epsilon\right.$ or $\left.1.5 \epsilon\right)$ melt states prepared with the PMD and DBH methods. These results are for $N_{c h}=500$ chains of length $N=500$, so finite-size effects are small [4]. The measured entanglement lengths depend dramatically on equilibration procedure. For states prepared using the DBH method, our results for $N_{e}$ agree with values from Ref. [3]. $N_{e}$ is smaller by a factor of two to three for the poorly-equilibrated, PMD-prepared states.

Another difference we find is that $\Delta b_{p p}$ is larger for poorly equilibrated initial states. Fluctuations in $b_{p p}$ indicate that friction between chains has prevented stress equilibration along the chains. This alone would not change the value of $N_{e}$ from Eqn. (7). However, $N_{e}$ would be decreased if friction prevented the chains from minimizing the total contour length, for example by trapping free loops along chains.
TABLE I: Primitive path analysis results for flexible and semiflexible melts - standard procedure. All of the PMD states listed were equilibrated for $480 \tau_{L J}$ following the fast pushoff. The quoted uncertainties in $N_{e}$ are the errors on the means of the distributions of $R_{e e}^{2} /\left((N-1) b_{p p}^{2}\right)$ for the $N_{c h}$ chains, with each chain considered an independent measurement.

\begin{tabular}{lcccc}
\hline \hline Method & $k_{\text {bend }} / \epsilon$ & $N_{e}$ & $<b_{p p}>/ a$ & $\Delta b_{p p} / a$ \\
DBH & 0 & $73.2 \pm 2.4$ & 0.1543 & 0.0037 \\
PMD & 0 & $24.9 \pm 0.9$ & 0.2695 & 0.0144 \\
DBH & 0.75 & $45.1 \pm 1.5$ & 0.2135 & 0.0042 \\
PMD & 0.75 & $18.3 \pm 0.6$ & 0.3397 & 0.0304 \\
DBH & 1.5 & $28.1 \pm 1.0$ & 0.3078 & 0.0119 \\
PMD & 1.5 & $13.1 \pm 0.5$ & 0.4510 & 0.0612 \\
\hline \hline
\end{tabular}

To test the potential magnitude of such effects we explored different algorithms for obtaining the primitive paths. These included beginning the PPA procedure at $T=1.0 \epsilon / k_{B}$ and cooling slowly, reducing the excluded volume interaction between adjacent monomers gradually, and increasing the value of $a$ in the LJ potential to reduce friction between chains. Ref. [4] specifies $\Delta b_{p p}<.006 a$ as a criterion for convergence of PPA runs to a state with uniform bond tension in the individual chains. For the standard PPA procedure, this convergence failed to occur for four of the six systems in Table II Table II shows results for these states with our altered PPA procedure. While improved stress equilibration reduces $\Delta b_{p p}$ by a factor of two, the values of $N_{e}$ increase by at most five percent. Thus it seems interchain friction is not responsible for the large differences in $N_{e}$.

TABLE II: Primitive path analysis results for flexible and semiflexible melts with procedure modified to reduce friction. Initial states and uncertainties are as in Table 凹

\begin{tabular}{lcccc}
\hline \hline Method & $k_{\text {bend }} / \epsilon$ & $N_{e}$ & $<b_{p p}>/ a$ & $\Delta b_{p p} / a$ \\
PMD & 0 & $26.1 \pm 0.9$ & 0.2638 & 0.0072 \\
PMD & 0.75 & $18.9 \pm 0.6$ & 0.3346 & 0.0143 \\
DBH & 1.5 & $28.8 \pm 1.0$ & 0.3040 & 0.0067 \\
PMD & 1.5 & $13.3 \pm 0.5$ & 0.4478 & 0.0286 \\
\hline \hline
\end{tabular}

The entanglement length of a bulk polymeric system is often related to large-scale measures of chain structure such as the packing length p [5]:

$$
p=\frac{N}{\rho<R_{e e}^{2}>},
$$

which is the volume occupied by a chain divided by its mean-square end-end distance. Extensive experiments on linear, Gaussian-chain homopolymers have shown that $N_{e} \propto \rho p^{3}$ [5, 6, 7]. The packing lengths are .68a and $.65 a$ for the DBH-prepared and PMD-prepared $k_{\text {bend }}=0$ states, respectively. Based on the experimentally observed power-law dependence, one might expect that 
the $N_{e}$ for the two systems should differ by only about $10-15 \%$. The actual difference in the $N_{e}$ measured by PPA analyses is a factor of three, as noted above. Similarly, the differences in $N_{e}$ between well- and poorlyequilibrated states for $k_{\text {bend }}=0.75 \epsilon$ and $k_{\text {bend }}=1.5 \epsilon$ are far too large to be explained by any difference in their large-scale structure.

The differences in $N_{e}$ may, however, be partially understood in terms of differences in short-scale structure. Figure 1 contrasts the chain statistics for the PMD and DBH flexible melt states, prior to primitive path analysis. To characterize chain configurations, we find it useful to define the Kuhn-length-like quantity

$$
l_{k}(n) \equiv \frac{<R^{2}(n)>}{n l_{0}} .
$$

This quantity was found to be more sensitive to equilibration than the other quantities considered in Ref. [10]. In Figure $1 l_{k}(n)$ for the DBH-prepared state increases nearly monotonically with $\mathrm{n}$, displaying a Gaussian limit $l_{k}(n) \simeq l_{K}=1.84 a$ for chemical distances $n>100$. The PMD states show pronounced non-Gaussian behavior. Results for $l_{k}(n)$ rise too steeply at small $\mathrm{n}$, reach a peak at $n=n_{\max }$, then drop to essentially the same large scale value as the $\mathrm{DBH}$ state as $n \rightarrow N[10]$. The differences in the short-scale chain configurations are qualitatively consistent with the difference in $N_{e}$; the straightening of chains at small scales in the PMD state is similar to that produced by increasing $k_{\text {bend }}$, which also lowers $N_{e}$ 5, 13,20$]$.

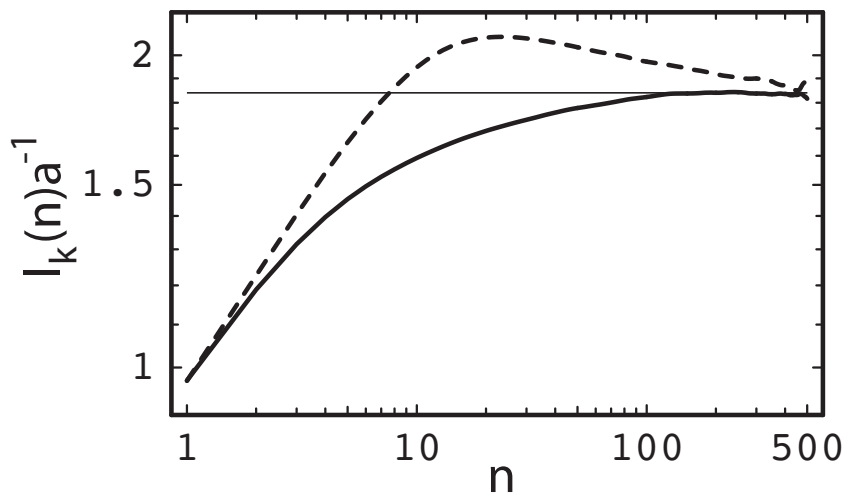

FIG. 1: Chain statistics $l_{k}(n)$ for PMD (dashed curve) and DBH (solid curve) melt states of 500 flexible chains of length $\mathrm{N}=500$. The horizontal line, $l_{k}(n)=1.84 a$, illustrates the large-n Gaussian limit of the well-equilibrated state.

Figure 2 contrasts the chain statistics of the primitive paths for the PMD and DBH flexible melt states. The peak in the PMD statistics at intermediate range is suppressed and the final curve is consistent with Gaussian statistics. This is why we get the same $N_{e}$ from Eqn. (7) or a fit to the full curve. The change in the PMD statistics during implementation of the PPA appears to be more dramatic than that for the DBH state. However, the relative displacement of monomers at $n>N_{e}$ is only of order $a$, i.e. small compared to the tube diameter, in both cases.

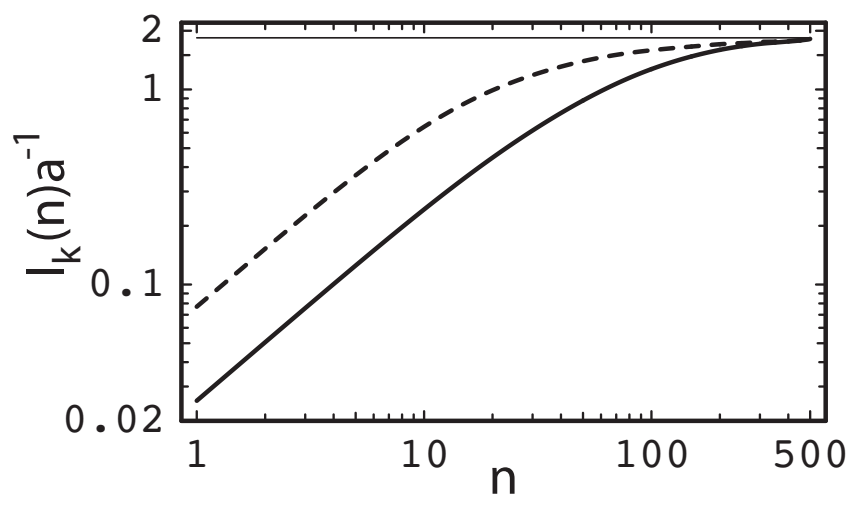

FIG. 2: Chain statistics $l_{k}(n)$ for PMD (dashed curve) and DBH (solid curve) melt states of 500 flexible chains of length $\mathrm{N}=500$, after implementation of the PPA.

\section{B. Evolution of $N_{e}$ with PMD Equilibration Time}

The effect of short-scale structure on $N_{e}$ can be further examined by following both during sample equilibration. Figure 3] shows how the melt chain statistics evolve with increasing PMD equilibration time for a semiflexible $\left(k_{\text {bend }}=0.75 \epsilon\right) N_{c h}=200, N=350$ system. After equilibration runs of order $10^{3} \tau_{L J}$ following the fast pushoff, the chain statistics show the short scale stretching typical of poorly equilibrated states [10]. After an equilibration run of one Rouse time, $\tau_{R} \sim 2 \cdot 10^{5} \tau_{L J}$ [12], the chain statistics are well equilibrated at chemical distances up to about ten monomers, but still out of equilibrium at chemical distances approaching the chain length. Table III shows additional information on the pre-PPA melt chain statistics, as well as PPA results for this system for PMD equilibration times up to $4.5 \cdot 10^{5} \tau_{L J} \sim 2 \tau_{R}$. Results for well-equilibrated DBH-prepared states from Ref. 3] are shown for comparison. The value of $N_{e}$ rises rapidly, doubling by $\tau_{R} / 4$ and tripling by $\sim \tau_{R}$. Note that the values of $\Delta b_{p p}$ drop with increasing $t_{e q}$ over roughly the same timescale, indicating that friction effects diminish.

The changes in $N_{e}$ and $l_{k}$ described above are much faster than the disentanglement time; $\tau_{R}$ is only about $\tau_{d} / 5$ for this system [12]. Thus the loss of entanglements must occur through a mechanism which is faster than reptation. Chain retraction is a mechanism studied in nonlinear-response tube theories [2] wherein the unentangled ends of a stretched chain contract rapidly inwards along its tube, thereby shortening the tube and releasing entanglement constraints. This process is much faster than reptation for $N / N_{e} \gg 1$ because motion of the chain ends arises from entropic tension rather than stochastic diffusion, and because the chain center of mass need not move 2]. Since the fast pushoff stretches chains, chain 
TABLE III: Evolution of PPA results for a $N_{c h}=200, N=350$ semiflexible $\left(k_{b e n d}=0.75 \epsilon\right)$ system with PMD melt equilibration time $t_{e q}$, following a fast pushoff. The Rouse time of these chains is $\tau_{R} \sim 2 \cdot 10^{5} \tau_{L J}$ 12]. Error bars are as defined in Table In the bottom row, $n_{\max }, l_{k}$, and $<R_{e e}^{2}>$ are limiting values for $N_{c h} \rightarrow \infty$, while $N_{e}, b_{p p}$, and $\Delta b_{p p}$ are from Ref. [3] .

\begin{tabular}{lccccc}
\hline \hline$t_{e q} / \tau_{L J}$ & $n_{\max }$ & $l_{k}\left(n_{\max }\right) / a$ & $10^{-2}<R_{e e}^{2}>/ a^{2}$ & $N_{e}$ & $<b_{p p}>/ a$ \\
$5 \cdot 10^{2}$ & 13 & 2.51 & 7.20 & $11 \pm 1$ & 0.434 \\
$3 \cdot 10^{3}$ & 18 & 2.39 & 6.89 & $12 \pm 2$ & 0.405 \\
$5 \cdot 10^{4}$ & 19 & 2.24 & 6.79 & $22 \pm 2$ & 0.300 \\
$1.0 \cdot 10^{5}$ & 21 & 2.16 & 6.22 & $25 \pm 2$ & 0.265 \\
$2.0 \cdot 10^{5}$ & 21 & 1.99 & 5.55 & $31 \pm 2$ & 0.048 \\
$3.0 \cdot 10^{5}$ & 26 & 2.04 & 5.82 & $34 \pm 3$ & 0.228 \\
$4.5 \cdot 10^{5}$ & 29 & 2.00 & 6.48 & $43 \pm 3$ & 0.220 \\
Equilibrated & 350 & 2.16 & 7.24 & $45 \pm 3$ & 0.207 \\
\hline \hline
\end{tabular}

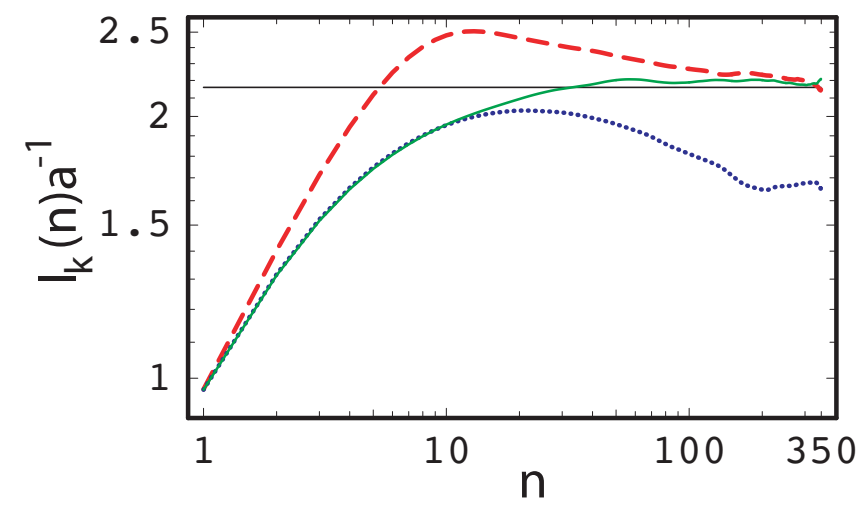

FIG. 3: Evolution of melt chain statistics $l_{k}(n)$ with PMD equilibration time for a 200-chain $\mathrm{N}=350$ system with $k_{\text {bend }}=0.75 \epsilon$ at $T=1.0 \epsilon / k_{B}$. The dashed and dotted curves are after $500 \tau_{L J}$ and $2 \cdot 10^{5} \tau_{L J}$ (about one Rouse time), respectively. The solid curve shows chain statistics of a wellequilibrated $\mathrm{DBH}$ state, and the thin horizontal line corresponds to the equilibrium Kuhn length, $l_{K}=2.16 a$.

retraction begins as soon as the Lennard-Jones interactions are activated. As shown in Figure 3 and Table III the chains rapidly contract at scales comparable to the end-end distance. $\left\langle R_{e e}^{2}\right\rangle$ drops as much as four standard deviations below its equilibrium value at $t_{e q} \simeq \tau_{R}$, showing that this contraction is not an equilibrium fluctuation.

As most of the chains are contracting in this way simultaneously, many topological constraints are also released far from chain ends. The combination of chain retraction and constraint release 2] accounts for the observed evolution of the chain statistics at small chemical distances. Another indication of the presence of constraint release is that the relaxation of the primitive path length $L_{p p}=(N-1)<b_{p p}>$, which is well fit by the tubetheory prediction for chain retraction [1]

$$
L_{p p}\left(t_{e q}\right)=L_{0}+\frac{L_{1}}{\sqrt{t_{e q}}}+L_{2} \sum_{\text {odd } \mathrm{p}} \frac{\exp \left(-p^{2} t_{e q} / \tau_{p p}\right)}{p^{2}}
$$

has a decay time which is clearly below $\tau_{R} ; \tau_{p p} \simeq 8$. $10^{4} \tau_{L J} \sim 0.4 \tau_{R}$. Studies with different initial states and $k_{\text {bend }}=0$ also show that entanglement loss is more rapid than reptation, with $\tau_{p p}$ about half of $\tau_{R}$.

As noted in Section III full equilibration of $N_{e}$ should occur only after the disentanglement time $\tau_{d}$, when the chains have vacated their original tubes. It is interesting that the $N_{e}=43$ value obtained for the longestequilibrated PMD state is consistent with values obtained for well-equilibrated states [3], when clearly the chain configurations remain far from equilibrium at large chemical distances. This suggests that short-range order plays the most important role in determining entanglements. Confirming this would require following the evolution of $N_{e}$ to $t>\tau_{d}$, which is beyond the scope of this study.

\section{Primitive Path Analyses of Glasses}

The six melt states from Table【were rapidly quenched, and standard primitive path analyses were applied to the resulting glassy states. In every case, the value of $N_{e}$ in the glass was close to that in the corresponding melt, even though melt values depended strongly on $k_{\text {bend }}$ and equilibration. It is often assumed [13, 14] that glasses inherit the melt value for $N_{e}$, and our results are consistent with this assumption to within statistical and systematic errors $(\sim 5 \%)$.

Nevertheless, it is interesting that the values of $N_{e}$ were lower in the glass than in the melt in all six cases. This reduction could in principle arise from an increase in interchain friction associated with the higher density of the glassy states, but the values of $\Delta b_{p p}$ in Table IV rule this out. All were remarkably close to the values for the corresponding melt states in Table $\llbracket$ indicating that friction is not responsible for the changes in $N_{e}$.

The small drop in $N_{e}$ from melt to glass might be associated with changes in chain statistics. An affine contraction of chains would not change $N_{e}$. However, while the density increases by $15 \%$ during the quench, the backbone bond length $l_{0}$ decreases by only $0.5 \%$. Chains in the glass are therefore stretched at short scales $(n<10)$ 
TABLE IV: Primitive path analysis results for glasses. The quoted uncertainties in $N_{e}$ (PPA) are as in Table 1 Values of $\lambda^{t h}$ use the PPA values of $N_{e}$, with $\lambda^{t h}=\sqrt{N_{e} l_{0} / l_{K}}$. Values of $\lambda$ are measured using the same procedure as Ref. [15], and $N_{e}^{t h}=\lambda^{2} l_{K} / l_{0}$.

\begin{tabular}{|c|c|c|c|c|c|c|}
\hline Method & $k_{\text {bend }} / \epsilon$ & $N_{e}(\mathrm{PPA})$ & $\Delta b_{p p} / a$ & $\lambda^{t h}$ & $\lambda$ & $N_{e}^{t h}$ \\
\hline $\mathrm{DBH}$ & 0 & $71.2 \pm 2.3$ & 0.0038 & $6.5 \pm 0.2$ & $5.9 \pm 0.4$ & $59 \pm 8$ \\
\hline PMD & 0 & $23.3 \pm 0.9$ & 0.0126 & $3.6 \pm 0.2$ & $5.9 \pm 0.2$ & $62 \pm 8$ \\
\hline $\mathrm{DBH}$ & 0.75 & $42.9 \pm 1.5$ & 0.0042 & $4.8 \pm 0.2$ & $5.4 \pm 0.8$ & $58 \pm 17$ \\
\hline PMD & 0.75 & $16.9 \pm 0.6$ & 0.0308 & $2.8 \pm 0.2$ & $5.2 \pm 0.4$ & $58 \pm 13$ \\
\hline $\mathrm{DBH}$ & 1.5 & $26.5 \pm 1.5$ & 0.0121 & $3.2 \pm 0.1$ & $3.7 \pm 0.4$ & $36 \pm 8$ \\
\hline PMD & 1.5 & $12.2 \pm 1.0$ & 0.0619 & $2.1 \pm 0.2$ & $4.1 \pm 0.3$ & $45 \pm 7$ \\
\hline
\end{tabular}

relative to an affine contraction from the melt. Reduction of $N_{e}$ due to this short-scale stretching would be consistent with our results for poorly equilibrated melts. Additional entanglements may be created as chain ends push outward during the quench.

Another possible explanation could be that the change in density couples to chain-thickness effects associated with the PPA. For states of constant density, increasing the bead diameter during implementation of the PPA decreases the calculated value of $N_{e}$. The values of $N_{e}$ in the glassy states average $94 \%$ of those in the corresponding melts, and $\left(\rho_{\text {glass }} / \rho_{\text {melt }}\right)^{-1 / 3}$ is also 0.94 . The increase in density from melt to glass could correspond to an increase in the effective chain thickness, which would be consistent with the observed reduction in $N_{e}$. We leave further discussion of this issue to a forthcoming paper.

Values of $N_{e}$ in polymer glasses are often inferred from measurements of the plateau shear modulus $\left(G^{0}=\right.$ $\left.4 \rho k_{B} T / 5 N_{e}\right)$ in the rubbery regime just above $T_{g}[13]$. Below the glass transition, rheological measurements become impossible, but $N_{e}$ can be measured via the craze extension ratio $\lambda=\rho_{0} / \rho_{\text {craze }}$ [14], where $\rho_{0}$ is the density of the undeformed glass and $\rho_{\text {craze }}$ is the density of a stable craze. The assumption that entanglements act like chemical crosslinks leads to the prediction $\lambda=\lambda^{t h} \equiv \sqrt{N_{e} l_{0} / l_{K}}$, where $l_{K}$ is the Kuhn length in the glass [14]. Experimental and simulation values for $\lambda$ at $T \ll T_{g}$ are consistent with this prediction for values of $N_{e}$ obtained from the plateau modulus [13, 15]. However, the prediction has not been tested for direct measurements of $N_{e}$ in the glassy state.

TableIV compares values of $\lambda^{t h}$ predicted by values of $N_{e}$ from PPA analysis of the undeformed glassy states to directly measured values of $\lambda$. The latter were obtained by straining uniaxially at constant velocity and measuring the ratio of the densities of coexisting uncrazed and crazed regions as in Ref. [15]. The values of $\lambda^{t h}$ and $\lambda$ are consistent for the DBH states, but not for PMD states [25]. Indeed, values of $\lambda$ are relatively insensitive to equilibration, which suggests that the craze extension ratio is controlled mainly by large-scale chain structure. Chains are greatly stretched as they pass from undeformed regions into the craze, and this may remove the memory of short-scale stretching from the fast pushoff.

\section{DISCUSSION AND CONCLUSIONS}

We have shown that the density of entanglements in a model polymeric melt can depend very strongly on equilibration of chain structure at short length scales. Values of the entanglement length for poorly equilibrated states prepared using non-crossing chain dynamics (PMD) were found to be as much as four times lower than values for well-equilibrated states prepared using an algorithm which changes chain connectivity (DBH). Coil-packing models, which focus on large-scale chain structure, fail to predict the magnitude of the differences in $N_{e}$. Instead, the low values of $N_{e}$ for poorly equilibrated states (Table I) can be attributed to local chain stretching caused by the fast pushoff procedure used to introduce excluded volume interactions.

At the conclusion of the fast pushoff, chain retraction ensues. Values of $N_{e}$ can increase rapidly towards apparent equilibrium over time scales comparable to the Rouse time as short-scale chain structure equilibrates (Table III Fig. 3). However, large-scale chain structure remains far from equilibrium at $\tau_{R}$, and $N_{e}$ may continue to evolve until the chains have vacated their original tubes after a time $\tau_{d}$. Since this time scales as $\left(N / N_{e}\right)^{3}$ for noncrossing chains, preparation methods which either entirely avoid producing the local chain stretching (e.g., through use of chain prepacking followed by a "slow" pushoff [10]), or accelerate equilibration by altering chain connectivity [10, 24], are far more suitable for the preparation of equilibrated states for primitive path analysis.

Changes in $N_{e}$ upon cooling from melts well above $T_{g}$ to glassy states well below $T_{g}$ were small for all equlibration protocols and chain stiffnesses examined. We did find that values of $N_{e}$ were uniformly lower in the glasses than in the melts; this could be attributed either to chainthickness effects in the implementation of the PPA or to the stiffness of backbone bonds relative to intermolecular bonds. However, the differences were comparable to the uncertainties in our measurements, and our results are consistent with the common assumption that the value of $N_{e}$ in glassy states is inherited from the melt. Finally, 
we found that values of $N_{e}$ inferred from measurements of the craze extension ratio were much less sensitive to equilibration than the values of $N_{e}$ obtained from PPA analyses of undeformed glassy states. This may indicate that deformation into the craze removes any memory of the short-scale chain stretching.

\section{ACKNOWLEDEGEMENTS}

The simulations in this paper were carried out using the LAMMPS molecular dynamics software (http://www.cs.sandia.gov/ sjplimp/lammps.html). Sathish K. Sukumaran, Jörg Rottler, Kurt Kremer, and especially Gary S. Grest provided initial states and helpful discussions. Support from NSF grant DMR-0454947 is gratefully acknowledged.
[1] M. Doi and S. F. Edwards, The Theory of Polymer Dynamics (Clarendon Press, Oxford, 1988).

[2] T. C. B. McLeish, Adv. Phys. 51, 1379 (2002).

[3] R. Everaers, S. K. Sukumaran, G. S. Grest, C. Svaneborg, A. Sivasubramanian, and K. Kremer, Science 303, 823 (2004).

[4] S. K. Sukumaran, G. S. Grest, K. Kremer, and R. Everaers, J. Polym. Sci. Part B - Polymer Physics 43, 917 (2005).

[5] L. J. Fetters, D. J. Lohse, D. Richter, T. A. Witten, and A. Zirkel, Macromolecules 27, 4639 (1994).

[6] L. J. Fetters, D. J. Lohse, and W. W. Graessley, J. Polym. Sci. Part B - Polymer Physics 37, 1023 (1999).

[7] L. J. Fetters, D. J. Lohse, S. T. Milner, and W. W. Graessley, Macromolecules 32, 6847 (1999).

[8] R. H. Colby, L. J. Fetters, W. G. Funk, and W. W. Graessley, Macromolecules 24, 3873 (1991).

[9] T. Inoue, Y. Yamashita, and K. Osaki, Macromolecules 35, 9169 (2002).

[10] R. Auhl, R. Everarers, G. S. Grest, K. Kremer, and S. J. Plimpton, J. Chem. Phys. 119, 12718 (2003).

[11] Q. Zhou and R. G. Larson, Macromolecules 38, 5761 (2005).

[12] M. Pütz, K. Kremer, and G. S. Grest, Europhys. Lett. 49, 735 (2000).

[13] R. N. Haward and R. J. Young, eds., The Physics of Glassy Polymers (Chapman \& Hall, London, 1997).
[14] E. J. Kramer and L. L. Berger, Adv. Polymer Sci. 91, 1 (1990).

[15] J. Rottler and M. O. Robbins, Phys. Rev. E 68, 011801 (2003).

[16] M. Bishop, D. Ceperley, H. L. Frisch, and M. H. Kalos, J. Chem. Phys. 72, 3229 (1980).

[17] K. Kremer and G. S. Grest, J. Chem. Phys. 92, 5057 (1990).

[18] D. Frenkel and B. Smit, Understanding Molecular Simulations, 2nd edition (Academic Press (San Diego), 2002).

[19] T. Schneider and E. Stoll, Phys. Rev. B 17, 1302 (1978).

[20] R. Faller and F. Müller-Plathe, ChemPhysChem 2, 180 (2001).

[21] R. Faller, A. Kolb, and F. Müller-Plathe, Phys. Chem. Chem. Phys. 1, 2071 (1999).

[22] D. N. Theodorou and U. W. Suter, Macromolecules 18, 1467 (1985).

[23] D. Brown, J. H. R. Clarke, M. Okuda, and T. Yamazaki, J. Chem. Phys. 100, 6011 (1994).

[24] N. C. Karayiannis, V. G. Mavrantzas, and D. N. Theodorou, Phys. Rev. Lett. 88, 105503 (2002).

[25] Our values of $\lambda$ for the PMD states are slightly higher than those reported in Ref. 15] for the semiflexible-chain systems, perhaps because of a different technique used to measure $\rho_{0} / \rho_{\text {craze }}$. 